\title{
Rank-based tests for comparison of multiple endpoints among several populations*
}

\author{
Zhengbang Li, Aiyi LiU ${ }^{\dagger}, Z_{\text {haOhai Li, And Qizhai Li }{ }^{\ddagger} \S}$
}

We consider comparison of multiple endpoints among several independent populations. We extend O'Brien's and Huang et al.'s methods from comparison of two groups to two more groups, and propose three max type test statistics, $T_{1}$ based on normally distributed data, $T_{2}$ obtained from pairwise ranking, and $T_{3}$ derived from ranking of all populations. Numerical results show that all three test statistics maintain the desired type I error rates and achieve satisfactory power. When the normal assumption is justified, $T_{1}$ is slightly more powerful than $T_{2}$ and $T_{3}$. However, when the normal assumption is violated, $T_{2}$ and $T_{3}$ gain sizable power. All three tests have higher power than O'Brien's and Huang et al.'s methods using Bonferroni correction under the considered settings. The methods are exemplified using healthy eating index data from a study examining the conformance to dietary guideline.

KEYWORdS AND PHRASES: Max, Multidimensional outcomes, Rank-based statistics.

\section{INTRODUCTION}

Comparison of multidimensional outcomes among different populations is commonly encountered in biomedical research. For example, in Brunner et al.'s (2002a) peptide procalcitonin $(\mathrm{PCT})$ concentration study, investigators are interested in the differences in PCT values among three treatment groups. Another example is a study to investigate compliance with dietary guideline among children with Type 1 diabetes. One study interest is to look into whether the income groups (low, medium, and high) differ in dietary quality that consists of 9 healthy eating indexes.

When the comparison is made between two groups, multivariate ANOVA in Brunner et al. (2002b) and Hotelling's $T^{2}$ test can be used under the assumption that the data from a group is distributed according to a multivariate normal distribution. O'Brien (1984) and Huang et al. (2005)

${ }^{*}$ We thank Dr. Tonja Nansel for providing the dietary quality and nutrients intake data to exemplify the methods.

†Supported by the Intramural Research Program of the Eunice Kennedy Shriver National Institute of Child Health and Human Development (NICHD), National Institutes of Health (NIH), USA.

$\ddagger$ Corresponding author.

§Partially supported by NNSFC, No. 61134013.

proposed distribution-free approaches based on rank statistics that gain power when the directions of the differences in the outcomes are in the same direction. If the directions are different (e.g. some are negative, some are positive), their rank-sum type tests could lose power substantially as shown in Liu et al. (2010). Liu et al. (2010) subsequently proposed a max-rank test, which is robust against directional differences between groups under comparison.

For comparison of multiple endpoints among two or more populations, in this paper, we extend O'Brien's (1984), Huang et al.'s (2005) and Liu et al.'s (2010) rank-based test and develop three test statistics, all sharing a common feature as the maximum of a series of marginal test statistics. The first test statistic, $T_{1}$, is based on normally distributed data. The second statistic, $T_{2}$, is obtained from pairwise ranking, and the third test statistic, $T_{3}$, is derived from ranking of all populations. We derive the asymptotic joint distributions of the marginal test statistics and evaluate the performance of the proposed three tests via simulation studies and compare them with O'Brien's (1984) and Huang et al.'s (2005) methods, which are derived for comparison for two groups, under Bonferroni-correction. We exemplify the methods using healthy eating index data from a study examining the conformance to dietary guideline.

\section{METHOD}

\subsection{Notations}

Suppose that there are $r(r \geq 2)$ populations for comparison. Let $\left(X_{a 1}, X_{a 2}, \ldots, X_{a p}\right)^{\prime}$ be a $p$-dimensional outcome for the ath population, $a=1,2, \ldots, r$, and let the marginal distribution function of $X_{a s}$ be $F_{a s}^{o}(x)=\operatorname{Pr}\left(X_{a s}<\right.$ $x)+\frac{1}{2} \operatorname{Pr}\left(X_{a s}=x\right)$ for $a=1,2, \ldots, r, s=1,2, \ldots, p, x \in R$. Let $\theta(a, b, s)=\operatorname{Pr}\left(X_{a s}<X_{b s}\right)-\operatorname{Pr}\left(X_{a s}>X_{b s}\right), a<b \in$ $\{1,2, \ldots, r\}, s=1,2, \ldots, p$. The null hypothesis is

$$
H_{0}: \theta(a, b, s)=0, \quad a<b \in\{1,2, \ldots, r\}, s=1,2, \ldots, p .
$$

The alternative hypothesis is that there is a set of $(a, b, s)^{\prime} \in$ $\{1,2, \ldots, r\} \otimes\{1,2, \ldots, r\} \otimes\{1,2, \ldots, p\}$ with $a<b$ such that $\theta(a, b, s) \neq 0$. Denote the $n_{a}$ observations from the $a$ th population by $\left(x_{a 1 i}, x_{a 2 i}, \ldots, x_{a p i}\right)^{\prime}, i=1,2, \ldots, n_{a}, a=$ $1,2, \ldots, r$.

When investigating the asymptotic distributions of test statistics, we assume, throughout the paper, that $n_{a} /$ 
$\sum_{b=1}^{r} n_{b} \longrightarrow \eta_{a}$, a finite non-negative constant, $a=1,2, \ldots$, $r$, such that $\sum_{a=1}^{r} \eta_{a}=1$ when $\min \left\{n_{1}, n_{2}, \ldots, n_{r}\right\} \longrightarrow$ $\infty$.

\subsection{A test statistic with normally distributed data}

Assume that the observations are from a multivariate normal distribution, that is, $\left(X_{a 1}, X_{a 2}, \ldots, X_{a p}\right)^{\prime} \sim N\left(\mu_{a}, \Lambda_{a}\right)$, where $\mu_{a}$ is a $p$-dimensional vector and $\Lambda_{a}$ is a $p \times p$ nonnegative definite matrix in Genz (1993), $a=1,2, \ldots, r$. Define

$$
\begin{aligned}
\bar{X}_{a s .}= & \frac{1}{n_{a}} \sum_{i=1}^{n_{a}} x_{a s i}, \quad a=1,2, \ldots, r, s=1,2, \ldots, p . \\
Z= & \left(\bar{X}_{11 \cdot}-\bar{X}_{21 .}, \ldots, \bar{X}_{1 p \cdot}-\bar{X}_{2 p .}, \ldots,\right. \\
& \left.\bar{X}_{(r-1) 1 \cdot}-\bar{X}_{r 1 .}, \ldots, \bar{X}_{(r-1) p \cdot}-\bar{X}_{r p \cdot}\right)_{\frac{r(r-1) p}{2} \times 1}^{\prime} .
\end{aligned}
$$

We have

Theorem 2.1. Denote $\Lambda=W A W^{\prime}$, where

$$
\begin{aligned}
& A=\left(\begin{array}{cccc}
\frac{1}{n_{1}} \Lambda_{1} & O_{p} & \cdots & O_{p} \\
O_{p} & \frac{1}{n_{2}} \Lambda_{2} & \cdots & O_{p} \\
\vdots & \vdots & \ddots & \vdots \\
O_{p} & O_{p} & \cdots & \frac{1}{n_{r}} \Lambda_{r}
\end{array}\right)_{r p \times r p} \\
& W=\left(\begin{array}{c}
W_{1} \\
W_{2} \\
\vdots \\
W_{r-1}
\end{array}\right)_{\frac{r(r-1) p}{2} \times r p} \\
& W_{1}=\left(\begin{array}{ccccccc}
I_{p} & -I_{p} & O_{p} & O_{p} & O_{p} & \cdots & O_{p} \\
I_{p} & O_{p} & -I_{p} & O_{p} & O_{p} & \cdots & O_{p} \\
I_{p} & O_{p} & O_{p} & -I_{p} & O_{p} & \cdots & O_{p} \\
\vdots & \vdots & \vdots & \vdots & \vdots & \ddots & \vdots \\
I_{p} & O_{p} & O_{p} & O_{p} & O_{p} & \cdots & -I_{p}
\end{array}\right)
\end{aligned}
$$

with orders $(r-1) p \times r p$,

$$
W_{2}=\left(\begin{array}{ccccccc}
O_{p} & I_{p} & -I_{p} & O_{p} & O_{p} & \cdots & O_{p} \\
O_{p} & I_{p} & O_{p} & -I_{p} & O_{p} & \cdots & O_{p} \\
O_{p} & I_{p} & O_{p} & O_{p} & -I_{p} & \cdots & O_{p} \\
\vdots & \vdots & \vdots & \vdots & \vdots & \ddots & \vdots \\
O_{p} & I_{p} & O_{p} & O_{p} & O_{p} & \cdots & -I_{p}
\end{array}\right)
$$

with orders $(r-2) p \times r p, \ldots, W_{r-1}=\left(O_{p}, O_{p}, \ldots, O_{p}, I_{p}\right.$, $\left.-I_{p}\right)_{p \times r p}, I_{p}$ is a p-dimension identity matrix, and $O_{p}$ is a $p \times p$ matrix with all the elements 0 . Then under $H_{0}$, $Z \sim N(\mathbf{0}, \Lambda)$.

The proof of Theorem 2.1 is given in Appendix A.1. Based on Theorem 2.1, a statistic for testing $H_{0}$ is given by

$$
T_{1} \max _{\{a<b \in\{1,2, \ldots, r\} ; s=1, \ldots, p\}}\left\{\frac{\left|\bar{X}_{a s \cdot}-\bar{X}_{b s \cdot}\right|}{\sqrt{\widehat{\operatorname{Var}}\left(\bar{X}_{a s \cdot}-\bar{X}_{b s}\right)}}\right\},
$$

where $\widehat{\operatorname{Var}}\left(\bar{X}_{a s .}-\bar{X}_{b s .}\right)=\frac{1}{n_{a}-1} \sum_{i=1}^{n_{a}}\left(x_{a s i}-\bar{X}_{a s .}\right)\left(x_{a t i}-\right.$ $\left.\bar{X}_{a t}\right)+\frac{1}{n_{b}-1} \sum_{i=1}^{n_{b}}\left(x_{b s i}-\bar{X}_{b s}.\right)\left(x_{b t i}-\bar{X}_{b t}.\right)$, for $a=1,2, \ldots, r$, $s, t \in\{1,2, \ldots, p\}$, a consistent estimate of the variance.

\subsection{A test statistic based on pairwise ranking}

When the distributional assumptions (e.g. normality) are violated, nonparametric test statistics such as those based on ranks are preferable due to their robustness against these assumptions O'Brien's (1984), Huang et al.'s (2005) and Liu et al. (2010). We construct a test statistic based on ranks between two populations. For $a<b \in$ $\{1,2, \ldots, r\}, s \in\{1,2, \ldots, p\}$, we rank the $n_{a}+n_{b}$ observations, $x_{a s 1}, x_{a s 2}, \ldots, x_{a s n_{a}}$ and $x_{b s 1}, x_{b s 2}, \ldots, x_{b s n_{b}}$ and denote the midranks of $x_{a s i}$ and $x_{b s j}$ by $R_{a s i}^{(a, b)}$ and $R_{b s j}^{(a, b)}$, respectively, $i=1, \ldots, n_{a}, j=1, \ldots, n_{b}$. Then we calculate $\bar{R}_{a s .}^{(a, b)}=\frac{1}{n_{a}} \sum_{i=1}^{n_{a}} R_{a s i}^{(a, b)}, \bar{R}_{b s .}^{(a, b)}=\frac{1}{n_{b}} \sum_{j=1}^{n_{b}} R_{b s j}^{(a, b)}, a<b \in$ $\{1,2, \ldots, r\}, s \in\{1,2, \ldots, p\}$. Define

$$
\begin{aligned}
U_{s}^{(a, b)}= & \bar{R}_{b s .}^{(a, b)}-\bar{R}_{a s}^{(a, b)} \\
= & \frac{n_{a}+n_{b}}{2 n_{a} n_{b}} \sum_{i=1}^{n_{a}} \sum_{j=1}^{n_{b}}\left[I_{\left\{x_{a s i}<x_{b s j}\right\}}-I_{\left\{x_{a s i}>x_{b s j}\right\}}\right], \\
& a<b \in\{1,2, \ldots, r\}, s=1,2, \ldots, p
\end{aligned}
$$

and

$U=\left(U_{1}^{(1,2)}, \ldots, U_{p}^{(1,2)}, \ldots, U_{1}^{(r-1, r)}, \ldots, U_{p}^{(r-1, r)}\right)_{\frac{r(r-1) p}{2} \times 1}^{\prime}$

We have

Theorem 2.2. Let

$$
B=\left(\begin{array}{cccc}
\frac{1}{\sqrt{n_{1}+n_{2}}} I_{p} & O_{p} & \cdots & O_{p} \\
O_{p} & \frac{1}{\sqrt{n_{1}+n_{3}}} I_{p} & \cdots & O_{p} \\
\vdots & \vdots & \ddots & \vdots \\
O_{p} & O_{p} & \cdots & \frac{1}{\sqrt{n_{r-1}+n_{r}}} I_{p}
\end{array}\right)
$$

and $\Delta$ be a $\frac{r(r-1) p}{2} \times \frac{r(r-1) p}{2}$ symmetric matrix, with upper elements given by

$$
\left(\begin{array}{cccc}
\Delta^{(12,12)} & \Delta^{(12,12)} & \cdots & \Delta^{(12,(r-1) r)} \\
& \Delta^{(13,13)} & \cdots & \Delta^{(13,(r-1) r)} \\
* & & \ddots & \vdots \\
& & & \Delta^{((r-1) r,(r-1) r)}
\end{array}\right)
$$

where $\Delta^{(a b, c d)}=\left(\delta^{(a b, c d)}(s, t)\right)_{p \times p}, a<b \in\{1,2, \ldots, r\}$, $c<d \in\{1,2, \ldots, r\}, s, t \in\{1,2, \ldots, p\}$ is a $p \times p$ symmetric matrix with elements as follows: 
when $a=c, b=d$,

$$
\begin{aligned}
\delta^{(a b, c d)}(s, t)= & \frac{\eta_{a}+\eta_{b}}{\eta_{a} \eta_{b}}\left[\eta_{b} \operatorname{cov}\left(F_{b s}^{o}\left(X_{a s}\right), F_{b t}^{o}\left(X_{a t}\right)\right)\right. \\
& \left.+\eta_{a} \operatorname{cov}\left(F_{a s}^{o}\left(X_{b s}\right), F_{a t}^{o}\left(X_{b t}\right)\right)\right]
\end{aligned}
$$

when $a=c, b \neq d$,

$$
\begin{aligned}
& \delta^{(a b, c d)}(s, t) \\
& =\frac{\sqrt{\left(\eta_{a}+\eta_{b}\right)\left(\eta_{a}+\eta_{d}\right)}}{\eta_{a}} \operatorname{cov}\left(F_{b s}^{o}\left(X_{a s}\right), F_{d t}^{o}\left(X_{a t}\right)\right) ;
\end{aligned}
$$

when $a \neq c, b=d$,

$$
\begin{aligned}
& \delta^{(a b, c d)}(s, t) \\
& =\frac{\sqrt{\left(\eta_{a}+\eta_{b}\right)\left(\eta_{c}+\eta_{b}\right)}}{\eta_{b}} \operatorname{cov}\left(F_{a s}^{o}\left(X_{b s}\right), F_{c t}^{o}\left(X_{b t}\right)\right) ;
\end{aligned}
$$

when $a \neq c, b \neq d, b=c$,

$$
\begin{aligned}
& \delta^{(a b, c d)}(s, t) \\
& \quad=-\frac{\sqrt{\left(\eta_{a}+\eta_{b}\right)\left(\eta_{b}+\eta_{d}\right)}}{\eta_{b}} \operatorname{cov}\left(F_{a s}^{o}\left(X_{b s}\right), F_{d t}^{o}\left(X_{b t}\right)\right) ;
\end{aligned}
$$

when $a \neq c, b \neq d, b \neq c, \delta^{(a b, c d)}(s, t)=0$.

Then under $H_{0}, B U \stackrel{\text { dist. }}{\longrightarrow} N(0, \Delta)$.

The proof of Theorem 2.2 is given in Appendix A.2 and the consistent estimates of the element of $\operatorname{cov}\left(F_{a s}^{o}\left(X_{b s}\right), F_{a t}^{o}\left(X_{b t}\right)\right), \operatorname{cov}\left(F_{a s}^{o}\left(X_{b s}\right), F_{c t}^{o}\left(X_{b t}\right)\right), \ldots$, and $\operatorname{cov}\left(F_{a s}^{o}\left(X_{b s}\right), F_{d t}^{o}\left(X_{b t}\right)\right)$ are given in Appendix A.4. Based on Theorem 2.2, we obtain a statistic for testing $H_{0}$ :

$$
T_{2}=\max _{\{a<b \in\{1,2, \ldots, r\}, s=1, \ldots, p\}}\left\{\left|U_{s}^{(a, b)}\right| / \sqrt{\widehat{\operatorname{Var}}\left(U_{s}^{(a, b)}\right)}\right\} .
$$

\subsection{A test statistic based on ranking among all populations}

In this section, we propose an alternative procedure based on ranks among all populations. We rank the $n\left(=\sum_{a=1}^{r} n_{a}\right)$ observations, $x_{1 s 1}, x_{1 s 2}, \ldots, x_{1 s n_{1}}, x_{2 s 1}, x_{2 s 2}$, $\ldots, x_{2 s n_{2}}, x_{r s 1}, x_{r s 2}, \ldots, x_{r s n_{r}}$, and denote the midranks of $x_{a s i}$ by $R_{a s i}, i=1, \ldots, n_{a}, a \in\{1, \ldots, r\}, s \in\{1, \ldots, p\}$.

Let

$$
\begin{aligned}
& R_{a s .}=\sum_{i=1}^{n_{a}} R_{a s i}, \quad \bar{R}_{a s .}=\frac{1}{n_{a}} R_{a s .}=\frac{1}{n_{a}} \sum_{i=1}^{n_{a}} R_{a s i}, \\
& R=\left(\bar{R}_{11}, \ldots, \bar{R}_{1 p .}, \bar{R}_{21 .}, \ldots, \bar{R}_{2 p .}, \ldots, \bar{R}_{r 1 .}, \ldots, \bar{R}_{r p .}\right)^{\prime},
\end{aligned}
$$

and

$$
\begin{array}{r}
G=\left(\bar{R}_{11 .}-\bar{R}_{21 .}, \bar{R}_{12 \cdot}-\bar{R}_{22 .}, \ldots, \bar{R}_{1 p \cdot}-\bar{R}_{2 p},\right. \\
\bar{R}_{11 .}-\bar{R}_{31 .}, \bar{R}_{12 .}-\bar{R}_{32 .}, \ldots, \bar{R}_{1 p \cdot}-\bar{R}_{3 p},
\end{array}
$$

$$
\begin{aligned}
& \bar{R}_{11 .}-\bar{R}_{r 1 .}, \bar{R}_{12 .}-\bar{R}_{r 2 .}, \ldots, \bar{R}_{1 p \cdot}-\bar{R}_{r p}, \\
& \bar{R}_{21} .-\bar{R}_{31}, \bar{R}_{22 .}-\bar{R}_{32 .}, \ldots, \bar{R}_{2 p} .-\bar{R}_{3 p}, \\
& \bar{R}_{21 .}-\bar{R}_{41 .}, \bar{R}_{22 .}-\bar{R}_{42 .}, \ldots, \bar{R}_{2 p} .-\bar{R}_{4 p} \text {, } \\
& \bar{R}_{21 .}-\bar{R}_{r 1 .}, \bar{R}_{22 .}-\bar{R}_{r 2 .}, \ldots, \bar{R}_{2 p .}-\bar{R}_{r p}, \\
& \bar{R}_{(r-1) 1 .}-\bar{R}_{r 1 .}, \bar{R}_{(r-1) 2 .}-\bar{R}_{r 2 .}, \ldots, \\
& \left.\bar{R}_{(r-1) p}-\bar{R}_{r p} \cdot\right)_{\frac{r(r-1) p}{2} \times 1}^{\prime} .
\end{aligned}
$$

We have

Theorem 2.3. Let $H^{\prime}=\left(\frac{n+1}{2}, \frac{n+1}{2}, \ldots, \frac{n+1}{2}\right)_{r p \times 1}$ and $V$ be a symmetric matrix whose upper elements are given by

$$
V=\left(\begin{array}{cccc}
V^{(1,1)} & V^{(1,2)} & \cdots & V^{(1, r)} \\
& V^{2,2} & \cdots & V^{(2, r)} \\
& * & \ddots & \vdots \\
& & & V^{(r, r)}
\end{array}\right)_{r p \times r p},
$$

where $V^{(a, b)}=\left(v^{(a, b)}(s, t)\right)_{p \times p}, a \leq b \in\{1,2, \ldots, r\}, s, t \in$ $\{1,2, \ldots, p\}$ is a $p \times p$ symmetric matrix with elements as follows:

when $a=b$,

$$
\begin{aligned}
v^{(a, b)}(s, t) & =\sum_{c=1, c \neq a}^{r}\left[\frac{\eta_{c}^{2}}{\eta_{a}} \operatorname{cov}\left(F_{c s}^{o}\left(X_{a s}\right), F_{c t}^{o}\left(X_{a t}\right)\right)\right. \\
& \left.+\eta_{c} \operatorname{cov}\left(F_{a s}^{o}\left(X_{c s}\right), F_{a t}^{o}\left(X_{c t}\right)\right)\right] \\
& +\sum_{c=1, c \neq a}^{r} \sum_{d=1, d \neq c, d \neq a}^{r} \frac{\eta_{c} \eta_{d}}{\eta_{a}} \operatorname{cov}\left(F_{c s}^{o}\left(X_{a s}\right), F_{d t}^{o}\left(X_{a t}\right)\right)
\end{aligned}
$$

when $a<b$,

$$
\begin{aligned}
v^{(a, b)}(s, t) & \\
= & -\left[\eta_{b} \operatorname{cov}\left(F_{b s}^{o}\left(X_{a s}\right), F_{b t}^{o}\left(X_{a t}\right)\right)\right. \\
& \left.+\eta_{a} \operatorname{cov}\left(F_{a s}^{o}\left(X_{b s}\right), F_{a t}^{o}\left(X_{b t}\right)\right)\right] \\
& +\sum_{c=1, c \neq a, c \neq b}^{r} \eta_{c}\left[\operatorname{cov}\left(F_{a s}^{o}\left(X_{c s}\right), F_{b t}^{o}\left(X_{c t}\right)\right)\right. \\
& \left.-\operatorname{cov}\left(F_{a s}^{o}\left(X_{b s}\right), F_{c t}^{o}\left(X_{b t}\right)\right)\right] .
\end{aligned}
$$

Then under $H_{0}$, we have

I) $E\left(R_{a s .}\right)=\frac{n_{a}(n+1)}{2}$ and $E\left(\bar{R}_{a s}\right)=\frac{n+1}{2}, a=1,2, \ldots, r$, $s=1,2, \ldots, p$.

II) $\frac{R-H}{\sqrt{n}} \stackrel{\text { dist. }}{\longrightarrow} N(0, V)$.

III) $\frac{G}{\sqrt{n}} \stackrel{\text { dist. }}{\longrightarrow} N\left(0, W V W^{\prime}\right)$, where $W$ is the same as in Theorem 2.1. 
The proof of Theorem 2.3 is given in Appendix A.3, and the consistent estimates of $\operatorname{cov}\left(F_{a s}^{o}\left(X_{b s}\right), F_{a t}^{o}\left(X_{b t}\right)\right)$, $\operatorname{cov}\left(F_{a s}^{o}\left(X_{b s}\right), F_{c t}^{o}\left(X_{b t}\right)\right), \ldots, \quad$ and $\operatorname{cov}\left(F_{a s}^{o}\left(X_{b s}\right), F_{d t}^{o}\left(X_{b t}\right)\right)$ are given in Appendix A.4. Using Theorem 2.3, we construct the following test statistic:

$$
T_{3}=\max _{\{a<b \in\{1,2, \ldots, r\}, s=1, \ldots, p\}}\left\{\frac{\left|\bar{R}_{a s .}-\bar{R}_{b s \cdot}\right|}{\sqrt{\overline{\operatorname{Var}}\left(\bar{R}_{a s .}-\bar{R}_{b s .}\right)}}\right\} .
$$

\subsection{P-value calculation}

Without loss of generality, we assume that $\left(Y_{1}, Y_{2}, \ldots, Y_{k}\right)^{\prime}$ follows a multivariate normal distribution. Let $Y=\max \left\{\left|Y_{1}\right|,\left|Y_{2}\right|, \ldots,\left|Y_{k}\right|\right\}$. After getting the observation $(y)$ of $Y$, we can use the following formula to calculate the p-value,

$$
\text { p-value }=\operatorname{Pr}(Y \geq y)=1-\operatorname{Pr}(Y<y),
$$

where $\operatorname{Pr}(Y<y)$ is a multiple integration; the integrand is the multivariate normal density function, and the integration region is a hyper-rectangle. The integral can be easily calculated using Genz and Bretz's (2009) algorithm, which allows for covariance and variance matrix to be singular.

\section{SIMULATION STUDIES}

We conduct simulation studies to explore the performances of $T_{1}, T_{2}$ and $T_{3}$ on the basis of type $I$ error rate and power by comparing with O'Brien's (1984) and Huang et al.'s (2005) methods. Since O'Brien (1984) and Huang et al.'s (2005) methods are derived for comparison of two groups and our simulation settings have 3 groups, we use Bonferroni-correction for their methods. For convenience, we denote two test statistics of O'Brien (1984) by OB1 and OB2 and Huang et al. (2005) by H1 and H2. We set $p=4$ and $r=3$. Two scenarios are considered here: 4 -variate normal distributions and 4-variate laplace distributions. For the simulation we consider $n_{1}=n_{2}=n_{3} \in\{100,200,300\}$.

\subsection{Multivariate normal distribution}

We first generate data from 4-variate normal distributions. To be specific, $\left(X_{11 i}, X_{12 i}, X_{13 i}, X_{14 i}\right)^{\prime}, i=1,2, \ldots$, $n_{1}$, are from a multivariate normal distribution with zero mean vector and variance-covariance matrix $\left(v_{s t}\right)_{4 \times 4}$, where $v_{s s}=1$ for $s=1,2,3,4$ and $v_{s t}=0.25$ for $s \neq t \in\{1,2,3,4\} ;\left(X_{21 j}, X_{22 j}, X_{23 j}, X_{24 j}\right)^{\prime}, j=1,2, \ldots$, $n_{2}$, are from a multivariate normal distribution with zero mean vector and variance-covariance matrix $\left(v_{s t}\right)_{4 \times 4}$, where $v_{s s}=2$ for $s=1,2,3,4$ and $v_{s t}=1$ for $s \neq t \in\{1,2,3,4\}$; and $\left(X_{31 k}, X_{32 k}, X_{33 k}, X_{34 k}\right)^{\prime}, k=1,2, \ldots, n_{3}$, are from a multivariate normal distribution with zero mean vector and variance-covariance matrix $\left(v_{s t}\right)_{4 \times 4}$, where $v_{s s}=4$ for $s=1,2,3,4$ and $v_{s t}=2.25$ for $s \neq t \in\{1,2,3,4\}$. The power is obtained similarly under the same settings with two scenarios being considered. One is that the mean vectors $(0.1,0.1,0.1,0.1),(0,0,0,0)^{\prime}$ and $(-0.1,-0.1$, $-0.1,-0.1)^{\prime}$ are for $\left(X_{11 i}, X_{12 i}, X_{13 i}, X_{14 i}\right)^{\prime}, i=1,2, \ldots$, $n_{1},\left(X_{21 j}, X_{22 j}, X_{23 j}, X_{24 j}\right)^{\prime}, j=1,2, \ldots, n_{2}$ and $\left(X_{31 k}\right.$,
Table 1. Empirical type I error rates (\# replicates is 2,000)

\begin{tabular}{cccccccc}
\hline \hline$n$ & OB1 & OB2 & $\mathrm{H} 1$ & $\mathrm{H} 2$ & $T_{1}$ & $T_{2}$ & $T_{3}$ \\
\hline \multicolumn{7}{c}{ Same directions } \\
\hline 100 & 0.055 & 0.055 & 0.048 & 0.048 & 0.054 & 0.060 & 0.058 \\
200 & 0.052 & 0.052 & 0.046 & 0.046 & 0.050 & 0.055 & 0.054 \\
300 & 0.050 & 0.049 & 0.042 & 0.042 & 0.051 & 0.048 & 0.049 \\
\hline \multicolumn{7}{c}{ Different directions } \\
\hline 100 & 0.049 & 0.049 & 0.043 & 0.041 & 0.049 & 0.058 & 0.059 \\
200 & 0.046 & 0.046 & 0.039 & 0.039 & 0.037 & 0.048 & 0.048 \\
300 & 0.054 & 0.054 & 0.045 & 0.045 & 0.049 & 0.053 & 0.054 \\
\hline
\end{tabular}

OB1 means O'Brien's (1984) method 1 under Bonferroni correction. OB2 means O'Brien's (1984) method 2 under Bonferroni correction. H1 means HUANG et al.'s (2005) method 1 under Bonferroni correction. H2 means HUANG et al.'s (2005) method 2 under Bonferroni correction.

Table 2. Empirical power for 4-variates normal distribution(\# replicates is 2,000 )

\begin{tabular}{cccccccc}
\hline \hline$n$ & OB1 & OB2 & H1 & H2 & $T_{1}$ & $T_{2}$ & $T_{3}$ \\
\hline \multicolumn{7}{c}{ Same directions } \\
\hline 100 & 0.178 & 0.177 & 0.162 & 0.162 & 0.156 & 0.163 & 0.166 \\
200 & 0.305 & 0.304 & 0.273 & 0.273 & 0.256 & 0.247 & 0.254 \\
300 & 0.452 & 0.452 & 0.408 & 0.408 & 0.373 & 0.345 & 0.363 \\
\hline \multicolumn{7}{c}{ Differenct directions } \\
\hline 100 & 0.050 & 0.050 & 0.044 & 0.044 & 0.631 & 0.584 & 0.607 \\
200 & 0.049 & 0.049 & 0.042 & 0.042 & 0.957 & 0.932 & 0.950 \\
300 & 0.042 & 0.042 & 0.036 & 0.036 & 0.998 & 0.995 & 0.998 \\
\hline
\end{tabular}

OB1 means O'Brien's (1984) method 1 under Bonferroni correction. OB2 means O'Brien's (1984) method 2 under Bonferroni correction. H1 means HUANG et al.'s (2005) method 1 under Bonferroni correction. H2 means HUANG et al.'s (2005) method 2 under Bonferroni correction.

$\left.X_{32 k}, X_{33 k}, X_{34 k}\right)^{\prime}, k=1,2, \ldots, n_{3}$, respectively; and another is that the mean vectors $(0.25,-0.25,0,0),(0,0,0,0)^{\prime}$ and $(-0.25,0.25,0,0)^{\prime}$ are for $\left(X_{11 i}, X_{12 i}, X_{13 i}, X_{14 i}\right)^{\prime}, i=$ $1,2, \ldots, n_{1},\left(X_{21 j}, X_{22 j}, X_{23 j}, X_{24 j}\right)^{\prime}, j=1,2, \ldots, n_{2}$ and $\left(X_{31 k}, X_{32 k}, X_{33 k}, X_{34 k}\right)^{\prime}, k=1,2, \ldots, n_{3}$, respectively.

2,000 replicates are generated to calculate the empirical type I error rates and power. The first panel of Table 1 shows the empirical type I error rates and Table 2 shows the power. Comparing with the nominal significance level, 0.05, all seven tests can control the type I error rates at the nominal level. For example, when $n_{1}=n_{2}=n_{3}=200$, the empirical type I error rates of OB1, OB2, H1, H2, $T_{1}$, $T_{2}$ and $T_{3}$ are $0.052,0.052,0.046,0.046,0.050,0.055$, and 0.054 , respectively.

For the power, as expected, when the differences of the features are in the same direction, OB1, OB2, H1, H2 is a little bit powerful than the proposed three tests. However,as expected, the proposed three tests are extremely more powerful than OB1, OB2, H1, and $\mathrm{H} 2$ when the directions are different. The powers of $T_{1}, T_{2}$ and $T_{3}$ are comparable. For example, when $n_{1}=n_{2}=n_{3}=300$, the power of OB1, OB2, H1, H2, $T_{1}, T_{2}$ and $T_{3}$ are $0.042,0.042,0.036,0.036$, $0.998,0.995$, and 0.998 respectively. 
Table 3. Empirical power for 4-variates laplace distribution (\# replicates is 2,000)

\begin{tabular}{cccccccc}
\hline \hline$n$ & OB1 & OB2 & H1 & H2 & $T_{1}$ & $T_{2}$ & $T_{3}$ \\
\hline \multicolumn{7}{c}{ Same directions } \\
\hline 100 & 0.216 & 0.215 & 0.190 & 0.190 & 0.160 & 0.201 & 0.208 \\
200 & 0.397 & 0.395 & 0.357 & 0.355 & 0.260 & 0.320 & 0.335 \\
300 & 0.571 & 0.571 & 0.524 & 0.524 & 0.388 & 0.458 & 0.482 \\
\hline \multicolumn{7}{c}{ Different directions } \\
\hline 100 & 0.047 & 0.047 & 0.041 & 0.040 & 0.622 & 0.766 & 0.793 \\
200 & 0.054 & 0.054 & 0.047 & 0.047 & 0.957 & 0.995 & 0.997 \\
300 & 0.046 & 0.046 & 0.040 & 0.040 & 0.998 & 1.000 & 1.000 \\
\hline
\end{tabular}

OB1 means O'Brien's (1984) method 1 under Bonferroni correction. OB2 means O'Brien's (1984) method 2 under Bonferroni correction. H1 means HUANG et al.'s (2005) method 1 under Bonferroni correction. H2 means HUANG et al.'s (2005) method 2 under Bonferroni correction.

\subsection{Multivariate laplace distribution}

In this section, we generate data from multivariate laplace distributions. The mean vectors and variance-covariance matrices for evaluation of type I error rates and power are the same as that for the multivariate normal distributions.

Again, 2,000 replicates are obtained to calculate the empirical type I error rates and power. The second panels of Table 1 and Table 3 show the results. Comparing with the nominal significance level, 0.05 , all seven tests have adequate control over the type I error rates. For example, when $n_{1}=n_{2}=n_{3}=300$, the empirical type I error rates of OB1, OB2, H1, H2, $T_{1}, T_{2}$ and $T_{3}$ are $0.054,0.054,0.045$, $0.045,0.049,0.053$, and 0.054 , respectively.

For the power, as expected, when the differences of the features are in the same direction, OB1, OB2, H1, H2 is a little bit powerful than the proposed three tests. However,as expected, the proposed three tests are extremely more powerful than OB1, OB2, H1, and $\mathrm{H} 2$ when the directions are different. The powers of $T_{1}, T_{2}$ and $T_{3}$ are comparable. For example, when $n_{1}=n_{2}=n_{3}=200$, the power of OB1, OB2, H1, H2, $T_{1}, T_{2}$ and $T_{3}$ are $0.054,0.054,0.047,0.047$, $0.957,0.995$, and 0.997 , respectively.

\section{DIETARY QUALITY AND NUTRIENTS INTAKE}

Type I diabetes (T1D, diabetes mellitus type I or juvenile diabetes) is a common disease in children with many potential health complications such as frequent urination, thirst, weight loss, weakened vision, and irritability. It has been speculated that diet may play a role in the development of type I diabetes, by influencing gut flora, intestinal permeability, and immune function in the gut; wheat in particular has been shown to have a connection to the development of type I diabetes, although the relationship has not been well understood (Nansel, Gellar and Mcgill, 2008; Knip, 2009). Dietary intervention in children and adolescents with type I diabetes with the goal of increasing consumption of nutrientdense carbohydrate-containing foods (e.g. fruits, vegetables, whole grains and legumes) and improving metabolic outcomes is believed to help attain normal control of glycemic indexes. To examine the conformance to dietary guideline, 3 -day diet records were obtained from a total number of 754 children aged 8-18 years with type I diabetes. Dietary quality and nutrient intake were measured. Higher scores indicate greater conformance to dietary guidelines. Children's basic characteristic information, such as age, gender, number of adults living with the children, and family incomes were also collected.

Dietary quality is measured by healthy eating index (HEI). A higher HEI score indicates better eating behavior and dietary quality, and compliance to the dietary guideline. The total HEI score was obtained by summing the HEI subscales including total fruit (HEI-1), total vegetable (HEI2), dark green/orange vegetables and legume (HEI-3), total grains (HEI-4), whole grains (HEI-5), dairy (HEI-6), meat (HEI-7), oils (HEI-8) and saturated fat (HEI-9). One question of interest of the study is whether family incomes affect patient's eating behavior and dietary quality. Here we examine whether healthy eating index subscales are different among families with different family incomes (three groups, low, medium, and high). For each child, the HEI subscales are obtained by averaging over the three days.

The statistical description of the 9 indexes is given in Table 4 . The table indicates that dietary quality in most categories appears to be higher among families with higher income. This could reflect a fact that family incomes play a role in food selection. The P-values of OB1, OB2, H1, $\mathrm{H} 2, T_{1}, T_{2}$, and $T_{3}$ are $0.034,0.033,0.104,0.090,0.0064$, 0.0057 , and 0.0052 respectively, with $T_{1}, T_{2}$, and $T_{2}$ being more significant than OB1, OB2, H1, and $\mathrm{H} 2$.

\section{CONCLUSIONS}

Comparing the differences among several groups with multiple endpoints is often encountered in biomedical research. The existing approaches in the literatures mostly focus on the differences between two populations. In this work, we extended the results of O'Brien's (1984), Huang et al.'s (2005) and Liu et al.'s (2010) rank-based results and proposed three test statistics $T_{1}, T_{2}$ and $T_{3}$ according to whether the normal assumptions hold, differences between pairs of interest, or differences between all groups of interest. When $r=2$, the proposed tests are reduced to be Liu et al.'s (2010) tests. Numerical results demonstrate that the proposed methods are more powerful than O'Brien's (1984) and Huang et al.'s (2005) methods under the Bonferroni corrections when the differences of the outcomes are in different directions. Part of the loss in power of their methods might be due to Bonferroni correction.

From Theorem 2.2 and Theorem 2.3, one can also construct two test statistics similar to O'Brien (1984) and Huang et al. (2005) using the linear combinations of the 
Table 4. Mean, median, standard deviation (sd), minimum value (min) and maximum (max)

\begin{tabular}{|c|c|c|c|c|c|c|c|c|c|c|}
\hline & & HEI1 & HEI2 & HEI3 & HEI4 & HEI5 & HEI6 & HEI7 & HEI8 & HEI9 \\
\hline \multirow{5}{*}{ Low } & mean & 1.72 & 2.13 & 0.19 & 4.60 & 2.30 & 5.44 & $\begin{array}{l}7.63 \\
\end{array}$ & 6.42 & 3.84 \\
\hline & median & 1.39 & 1.75 & 0.00 & 5.00 & 1.91 & 5.27 & 9.64 & 6.74 & 3.12 \\
\hline & sd & 1.82 & 1.55 & 0.39 & 0.84 & 1.95 & 2.85 & 3.12 & 3.16 & 3.72 \\
\hline & $\min$ & 0.00 & 0.00 & 0.00 & 0.00 & 0.00 & 0.00 & 0.00 & 0.00 & 0.00 \\
\hline & $\max$ & 5.00 & 5.00 & 2.55 & 5.00 & 5.00 & 10.00 & 10.00 & 10.00 & 10.00 \\
\hline \multirow{5}{*}{ Med. } & mean & 2.00 & 2.20 & 0.11 & 4.67 & 2.37 & 5.22 & 7.39 & 6.83 & 4.55 \\
\hline & median & 1.65 & 1.89 & 0.00 & 5.00 & 2.18 & 5.15 & 9.12 & 7.84 & 4.92 \\
\hline & $\mathrm{sd}$ & 1.91 & 1.67 & 0.22 & 0.78 & 1.99 & 2.95 & 3.28 & 3.28 & 3.55 \\
\hline & $\min$ & 0.00 & 0.00 & 0.00 & 0.44 & 0.00 & 0.00 & 0.00 & 0.13 & 0.00 \\
\hline & $\max$ & 5.00 & 5.00 & 1.51 & 5.00 & 5.00 & 10.00 & 10.00 & 10.00 & 10.00 \\
\hline \multirow{5}{*}{ High } & mean & 2.09 & 2.33 & 0.20 & 4.75 & 2.76 & 5.61 & 7.31 & 6.61 & 4.40 \\
\hline & median & 1.76 & 2.10 & 0.00 & 5.00 & 3.10 & 5.30 & 8.60 & 6.90 & 4.21 \\
\hline & sd & 1.86 & 1.62 & 0.33 & 0.57 & 2.03 & 2.78 & 3.30 & 3.32 & 3.58 \\
\hline & $\min$ & 0.00 & 0.00 & 0.00 & 2.31 & 0.00 & 0.00 & 0.00 & 0.04 & 0.00 \\
\hline & $\max$ & 5.00 & 5.00 & 1.98 & 5.00 & 5.00 & 10.00 & 10.00 & 10.00 & 10.00 \\
\hline
\end{tabular}

test statistic for each outcome. However, as stated in Liu et al. (2010), the proposed max-type test statistic is a "global" test and can handle the different directions of the effects for the endpoints. So the proposed tests tend to be more robust than "linear"-type tests, which are constructed using linear combinations of the test statistic for each endpoint. On the other hand, after the significant results from such global test, one can use Liu et al.'s method or O'Brien's and Huang et al.'s approach to do the pairwise comparison and then find the differences of two groups.

\section{APPENDIX A. THEORIES AND ESTIMATES}

\section{A.1 Proof of Theorem 2.1}

Let $Y=\left(\bar{X}_{11}, \bar{X}_{12 .}, \ldots, \bar{X}_{1 p .}, \bar{X}_{21 .}, \bar{X}_{22} \ldots, \bar{X}_{2 p .}, \ldots\right.$, $\left.\bar{X}_{r 1 .}, \bar{X}_{r 2} \ldots, \bar{X}_{r p .}\right)^{\prime}, \mu=\left(\mu_{1}^{\prime}, \ldots, \mu_{r}^{\prime}\right)^{\prime}$. Then $Y \sim N(\mu, A)$ and $Z=W Y$. Then we have the result by applying a well known result in Tong (1990) to determine the covariance matrix of a linear transformation of multivariate normal variables.

\section{A.2 Proof of Theorem 2.2}

Since

$$
\begin{aligned}
U= & \left(\frac{\sqrt{n_{1}+n_{2}}}{2 n_{1} n_{2}} \sum_{i=1}^{n_{1}} \sum_{j=1}^{n_{2}}\left[I_{\left\{x_{11 i}<x_{21 j}\right\}}-I_{\left\{x_{11 i}>x_{21 j}\right\}}\right], \ldots,\right. \\
& \left.\frac{\sqrt{n_{r-1}+n_{r}}}{2 n_{r-1} n_{r}} \sum_{i=1}^{n_{r-1}} \sum_{j=1}^{n_{r}}\left[I_{\left\{x_{(r-1) p i}<x_{r p j}\right\}}-I_{\left\{x_{(r-1) p i}>x_{r p j}\right\}}\right]\right)^{\prime}
\end{aligned}
$$

is a U-statistic, it converges in distribution to a normal distribution as $\min \left\{n_{1}, n_{2}, \ldots, n_{r}\right\} \rightarrow \infty$.

We now calculate the variance and covariance matrix of $U$. For $a<b \in\{1,2, \ldots, r\}$ and $c<d \in\{1,2, \ldots, r\}$, denote

$$
\begin{aligned}
\Omega^{(a b, c d)} & \\
= & \operatorname{cov}\left(\left(\frac{U_{1}^{(a, b)}}{\sqrt{n_{a}+n_{b}}}, \frac{U_{2}^{(a, b)}}{\sqrt{n_{a}+n_{b}}}, \ldots, \frac{U_{p}^{(a, b)}}{\sqrt{n_{a}+n_{b}}}\right)^{\prime},\right. \\
& \left.\left(\frac{U_{1}^{(c, d)}}{\sqrt{n_{c}+n_{d}}}, \frac{U_{2}^{(c, d)}}{\sqrt{n_{c}+n_{d}}}, \ldots, \frac{U_{p}^{(c, d)}}{\sqrt{n_{c}+n_{d}}}\right)^{\prime}\right) \\
\hat{=} & \left(\delta^{(a b, c d)}(s t)\right)_{p \times p} .
\end{aligned}
$$

We have $\Omega^{(a b, a b)}=\Delta^{(a b, a b)}$ based on Theorem 1 in Liu et al. (2010) as $\min \left\{n_{1}, n_{2}, \ldots, n_{r}\right\} \rightarrow \infty$.

When $a=c, b \neq d$,

$\operatorname{cov}\left(I_{\left\{x_{a s i}<x_{b s k}\right\}}-I_{\left\{x_{a s i}>x_{b s k}\right\}}, I_{\left\{x_{a t j}<x_{d t m}\right\}}-I_{\left\{x_{a t j}>x_{d t m}\right\}}\right)=0$ for $i \neq j \in\left\{1, \ldots, n_{a}\right\}, k \in\left\{1, \ldots, n_{b}\right\}, m \in\left\{1, \ldots, n_{d}\right\}$,

and

$$
\begin{aligned}
\operatorname{cov}( & \left.I_{\left\{x_{a s i}<x_{b s k}\right\}}-I_{\left\{x_{a s i}>x_{b s k}\right\}}, I_{\left\{x_{a t i}<x_{d t m}\right\}}-I_{\left\{x_{a t i}>x_{d t m}\right\}}\right) \\
= & E\left\{E \left[( I _ { \{ x _ { a s i } < x _ { b s k } \} } - I _ { \{ x _ { a s i } > x _ { b s k } \} } ) \left(I_{\left\{x_{a t i}<x_{d t m}\right\}}\right.\right.\right. \\
& \left.\left.\left.-I_{\left\{x_{a t i}>x_{d t m}\right\}}\right)\left(x_{a s i}, x_{a t i}\right)\right]\right\} \\
= & E\left[\left[1-2 F_{b s}^{o}\left(X_{a s}\right)\right]\left[1-2 F_{d t}^{o}\left(X_{a t}\right)\right]\right] \\
= & 4 \operatorname{cov}\left(F_{b s}^{o}\left(X_{a s}\right), F_{d t}^{o}\left(X_{a t}\right)\right) \\
& \text { for } i \in\left\{1, \ldots, n_{a}\right\}, k \in\left\{1, \ldots, n_{b}\right\}, m \in\left\{1, \ldots, n_{d}\right\},
\end{aligned}
$$

where we use $E\left[F_{b s}^{o}\left(X_{a s}\right)\right]=1 / 2, a \neq b=1,2, \ldots, r, s=$ $1, \ldots, p$, under $H_{0}$.

It thus follows that

$$
\begin{aligned}
& \delta^{(a b, a d)}(s, t) \\
& \quad=\operatorname{cov}\left(\frac{U_{s}^{(a, b)}}{\sqrt{n_{a}+n_{b}}}, \frac{U_{t}^{(a, d)}}{\sqrt{n_{a}+n_{d}}}\right) \\
& =\operatorname{cov}\left(\frac{\sqrt{n_{a}+n_{b}}}{2 n_{a} n_{b}} \sum_{i=1}^{n_{a}} \sum_{k=1}^{n_{b}}\left(I_{\left\{x_{a s i}<x_{b s k}\right\}}-I_{\left\{x_{a s i}>x_{b s k}\right\}}\right),\right.
\end{aligned}
$$




$$
\begin{aligned}
& \left.\frac{\sqrt{n_{a}+n_{d}}}{2 n_{a} n_{d}} \sum_{j=1}^{n_{a}} \sum_{m=1}^{n_{d}}\left(I_{\left\{x_{a t j}<x_{d t m}\right\}}-I_{\left\{x_{a t j}>x_{d t m}\right\}}\right)\right) \\
= & \frac{\sqrt{\left(n_{a}+n_{b}\right)\left(n_{a}+n_{d}\right)}}{4 n_{a}^{2} n_{b} n_{d}} 4 n_{a} n_{b} n_{d} \operatorname{cov}\left(F_{b s}^{o}\left(X_{a s}\right), F_{d t}^{o}\left(X_{a t}\right)\right) \\
= & \frac{\sqrt{\left(n_{a}+n_{b}\right)\left(n_{a}+n_{d}\right)}}{n_{a}} \operatorname{cov}\left(F_{b s}^{o}\left(X_{a s}\right), F_{d t}^{o}\left(X_{a t}\right)\right) \\
= & \frac{\sqrt{\left(\eta_{a}+\eta_{b}\right)\left(\eta_{a}+\eta_{d}\right)}}{\eta_{a}} \operatorname{cov}\left(F_{b s}^{o}\left(X_{a s}\right), F_{d t}^{o}\left(X_{a t}\right)\right)
\end{aligned}
$$

as $\min \left\{n_{1}, \ldots, n_{r}\right\} \longrightarrow \infty$.

Hence $\Omega^{(a b, a d)}=\Delta^{(a b, a d)}$ as $\min \left\{n_{1}, \ldots, n_{r}\right\} \longrightarrow \infty$. When $a \neq c, b=d, \Omega^{(a b, c b)}=\Delta^{(a b, c b)}$ as $\min \left\{n_{1}, \ldots\right.$, $\left.n_{r}\right\} \longrightarrow \infty$ where the derivation is similar to above.

When $a \neq c, b \neq d, b=c$,

$\operatorname{cov}\left(I_{\left\{x_{a s k}<x_{b s i}\right\}}-I_{\left\{x_{a s k}>x_{b s i}\right\}}, I_{\left\{x_{b t j}<x_{d t m}\right\}}-I_{\left\{x_{b t j}>x_{d t m}\right\}}\right)=0$

for $i \neq j \in\left\{1, \ldots, n_{b}\right\}, k \in\left\{1, \ldots, n_{a}\right\}, m \in\left\{1, \ldots, n_{d}\right\}$

and

$$
\begin{aligned}
\operatorname{cov}( & \left.I_{\left\{x_{a s k}<x_{b s i}\right\}}-I_{\left\{x_{a s k}>x_{b s i}\right\}}, I_{\left\{x_{b t i}<x_{d t m}\right\}}-I_{\left\{x_{b t i}>x_{d t m}\right\}}\right) \\
= & E\left\{E \left[\left(I_{\left\{x_{a s k}<x_{b s i}\right\}}-I_{\left\{x_{a s k}>x_{b s i}\right\}}\right)\right.\right. \\
& \left.\left.\times\left(I_{\left\{x_{b t i}<x_{d t m}\right\}}-I_{\left\{x_{b t i}>x_{d t m}\right\}}\right) \mid\left(x_{b s i}, x_{b t i}\right)\right]\right\} \\
= & E\left[\left[2 F_{a s}^{o}\left(X_{b s}\right)-1\right]\left[1-2 F_{d t}^{o}\left(X_{b t}\right)\right]\right] \\
= & -4 \operatorname{cov}\left(F_{a s}^{o}\left(X_{b s}\right), F_{d t}^{o}\left(X_{b t}\right)\right) \\
& \text { for } i \in\left\{1, \ldots, n_{b}\right\}, k \in\left\{1, \ldots, n_{a}\right\}, m \in\left\{1, \ldots, n_{d}\right\} .
\end{aligned}
$$

Therefore,

$$
\begin{aligned}
\delta^{(a b, b d)}(s, t) & \operatorname{cov}\left(\frac{U^{(a, b)}}{\sqrt{n_{a}+n_{b}}}, \frac{U^{(b, d)}}{\sqrt{n_{b}+n_{d}}}\right) \\
= & \operatorname{cov}\left(\frac{\sqrt{n_{a}+n_{b}}}{2 n_{a} n_{b}} \sum_{k=1}^{n_{a}} \sum_{i=1}^{n_{b}}\left[I_{\left\{x_{a s k}<x_{b s i}\right\}}-I_{\left\{x_{a s k}>x_{b s i}\right\}}\right],\right. \\
& \frac{\left.\sqrt{n_{b}+n_{d}} \sum_{b} \sum_{j=1}^{n_{d}}\left[I_{\left\{x_{b t j}<x_{d t m}\right\}}-I_{\left\{x_{b t j}>x_{d t m}\right\}}\right]\right)}{2 n_{b} n_{d}} \sum_{j=1} \\
=- & \frac{\sqrt{\left(n_{a}+n_{b}\right)\left(n_{b}+n_{d}\right)}}{4 n_{b}^{2} n_{a} n_{d}} 4 n_{a} n_{b} n_{d} \operatorname{cov}\left(F_{a s}^{o}\left(X_{b s}\right), F_{d t}^{o}\left(X_{b t}\right)\right) \\
n_{b} & \operatorname{dn}\left(F_{a s}^{o}\left(X_{b s}\right), F_{d t}^{o}\left(X_{b t}\right)\right) \\
= & \frac{\sqrt{\left(\eta_{a}+\eta_{b}\right)\left(\eta_{b}+\eta_{d}\right)}}{\eta_{b}} \operatorname{cov}\left(F_{a s}^{o}\left(X_{b s}\right), F_{d t}^{o}\left(X_{b t}\right)\right)
\end{aligned}
$$

as $\min \left\{n_{1}, \ldots, n_{r}\right\} \longrightarrow \infty$, which indicates that $\Omega^{(a b, b d)}=$ $\Delta^{(a b, b d)}$ as $\min \left\{n_{1}, \ldots, n_{r}\right\} \longrightarrow \infty$. When $a \neq c, b \neq d, b \neq c$, $\operatorname{cov}\left(\frac{U^{(a, b)}}{\sqrt{n_{a}+n_{b}}}, \frac{U^{(c, d)}}{\sqrt{n_{c}+n_{d}}}\right)=0$.

\section{A.3 Proof of Theorem 2.3}

I) Denote the ordered statistic among $x_{a s 1}, x_{a s 2}, \ldots$, $x_{a s n_{a}}$ by $x_{a s(i)}, i=1,2, \ldots, n_{a}, a=1,2, \ldots, r$. Then

$$
\begin{aligned}
R_{a s .} & =\sum_{i=1}^{n_{a}}\left(i+\sum_{b=1, b \neq a}^{r} \sum_{j=1}^{n_{b}} I_{\left\{x_{b s j}<x_{a s(i)}\right\}}\right) \\
& =\frac{n_{a}\left(n_{a}+1\right)}{2}+\sum_{b=1, b \neq a}^{r} \sum_{j=1}^{n_{b}} \sum_{i=1}^{n_{a}} I_{\left\{x_{b s j}<x_{a s i}\right\}} .
\end{aligned}
$$

So,

$$
\begin{aligned}
& R_{a s \cdot}-\frac{n_{a}(n+1)}{2}=\sum_{b=1, b \neq a}^{r} \sum_{j=1}^{n_{b}} \sum_{i=1}^{n_{a}}\left(I_{\left\{x_{b s j}<x_{a s i}\right\}}-\frac{1}{2}\right), \\
& s=1,2, \ldots, p,
\end{aligned}
$$

and

$$
\begin{aligned}
& \bar{R}_{a s .}-\frac{n+1}{2}=\frac{1}{n_{a}} \sum_{b=1, b \neq a}^{r} \sum_{j=1}^{n_{b}} \sum_{i=1}^{n_{a}}\left(I_{\left\{x_{b s j}<x_{a s i}\right\}}-\frac{1}{2}\right) \\
& s=1,2, \ldots, p .
\end{aligned}
$$

Under $H_{0}$, we have the conclusion.

II) Since $R$ is a U-statistic, it converges to a normal distribution when $\min \left\{n_{1}, n_{2}, \ldots, n_{r}\right\} \rightarrow \infty$ under $H_{0}$. Denote the variance and covariance matrix of $\left(\frac{\bar{R}_{a 1 \cdot}-\frac{n+1}{2}}{\sqrt{n}}, \frac{\bar{R}_{a 2 \cdot}-\frac{n+1}{2}}{\sqrt{n}}\right.$, $\left.\ldots, \frac{\bar{R}_{a p \cdot}-\frac{n+1}{2}}{\sqrt{n}}\right)^{\prime}$ and $\left(\frac{\bar{R}_{b 1}-\frac{n+1}{2}}{\sqrt{n}}, \frac{\bar{R}_{b 2 \cdot}-\frac{n+1}{2}}{\sqrt{n}}, \ldots, \frac{\bar{R}_{b p \cdot}-\frac{n+1}{2}}{\sqrt{n}}\right)^{\prime}$ by $V^{(a, b)}=\left(v^{(a, b)}(s, t)\right)_{p \times p}$. We have

$$
\begin{aligned}
v^{(a, a)}(s, t) & \operatorname{cov}\left(\frac{1}{\sqrt{n} n_{a}} \sum_{c=1, c \neq a}^{r} \sum_{j=1}^{n_{c}} \sum_{i=1}^{n_{a}}\left(I_{\left\{x_{c s j}<x_{a s i}\right\}}-1 / 2\right),\right. \\
& \left.\frac{1}{\sqrt{n} n_{a}} \sum_{c=1, c \neq a}^{r} \sum_{m=1}^{n_{c}} \sum_{k=1}^{n_{a}}\left(I_{\left\{x_{c t m}<x_{a t k}\right\}}-1 / 2\right)\right) \\
= & \sum_{c=1, c \neq a}^{r} \operatorname{cov}\left(\frac{1}{\sqrt{n} n_{a}} \sum_{j=1}^{n_{c}} \sum_{i=1}^{n_{a}}\left(I_{\left\{x_{c s j}<x_{a s i}\right\}}-1 / 2\right),\right. \\
& \left.\frac{1}{\sqrt{n} n_{a}} \sum_{m=1}^{n_{c}} \sum_{k=1}^{n_{a}}\left(I_{\left\{x_{c t m}<x_{a t k}\right\}}-1 / 2\right)\right) \\
& +\sum_{c=1, c \neq a}^{r} \sum_{d=1, d \neq a, d \neq c}^{r} \operatorname{cov}\left(\frac{1}{\sqrt{n} n_{a}} \sum_{j=1}^{n_{c}} \sum_{i=1}^{n_{a}}\left(I_{\left\{x_{c s j}<x_{a s i}\right\}}-1 / 2\right),\right. \\
& \left.\frac{1}{\sqrt{n} n_{a}} \sum_{m=1}^{n_{d}} \sum_{k=1}^{n_{a}}\left(I_{\left\{x_{d t m}<x_{a t k}\right\}}-1 / 2\right)\right) \\
= & \sum_{c=1, c \neq a}^{r} \frac{1}{n n_{a}^{2}}\left[n_{a} n_{c}\left(n_{c}-1\right) \operatorname{cov}\left(F_{c s}^{o}\left(X_{a s}\right), F_{c t}^{o}\left(X_{a t}\right)\right)\right. \\
& \left.+n_{c} n_{a}\left(n_{a}-1\right) \operatorname{cov}\left(F_{a s}^{o}\left(X_{c s}\right), F_{a t}^{o}\left(X_{c t}\right)\right)+n_{a} n_{c} \sigma\right] \\
= &
\end{aligned}
$$




$$
\begin{aligned}
& +\sum_{c=1, c \neq a}^{r} \sum_{d=1, d \neq a, d \neq c}^{r} \frac{1}{n n_{a}^{2}} n_{a} n_{c} n_{d} \\
& \times \operatorname{cov}\left(F_{c s}^{o}\left(X_{a s}\right), F_{d t}^{o}\left(X_{a t}\right)\right),
\end{aligned}
$$

where $|\sigma| \leq 1$, and more details about $\sigma$ can refer to Liu et al. (2010). When $\min \left\{n_{1}, n_{2}, \ldots, n_{r}\right\} \rightarrow \infty$,

$$
\begin{aligned}
v^{(a, a)}(s, t) & =\sum_{c=1, c \neq a}^{r}\left[\frac{\eta_{c}^{2}}{\eta_{a}} \operatorname{cov}\left(F_{c s}^{o}\left(X_{a s}\right), F_{c t}^{o}\left(X_{a t}\right)\right)\right. \\
& \left.+\eta_{c} \operatorname{cov}\left(F_{a s}^{o}\left(X_{c s}\right), F_{a t}^{o}\left(X_{c t}\right)\right)\right] \\
& +\sum_{c=1, c \neq a}^{r} \sum_{d=1, d \neq a, d \neq c}^{r} \frac{\eta_{c} \eta_{d}}{\eta_{a}} \operatorname{cov}\left(F_{c s}^{o}\left(X_{a s}\right), F_{d t}^{o}\left(X_{a t}\right)\right) .
\end{aligned}
$$$$
\left.v^{(a, b)}(s, t)\right|_{a \neq b}
$$$$
=\operatorname{cov}\left(\frac{1}{\sqrt{n} n_{a}} \sum_{c=1, c \neq a}^{r} \sum_{j=1}^{n_{c}} \sum_{i=1}^{n_{a}}\left(I_{\left\{x_{c s j}<x_{a s i}\right\}}-1 / 2\right),\right.
$$$$
\left.\frac{1}{\sqrt{n} n_{b}} \sum_{d=1, d \neq b}^{r} \sum_{m=1}^{n_{d}} \sum_{k=1}^{n_{b}}\left(I_{\left\{x_{d t m}<x_{b t k}\right\}}-1 / 2\right)\right)
$$$$
=\operatorname{cov}\left(\frac{1}{\sqrt{n} n_{a}} \sum_{j=1}^{n_{b}} \sum_{i=1}^{n_{a}}\left(I_{\left\{x_{b s j}<x_{a s i}\right\}}-1 / 2\right)\right. \text {, }
$$$$
\left.\frac{1}{\sqrt{n} n_{b}} \sum_{m=1}^{n_{a}} \sum_{k=1}^{n_{b}}\left(I_{\left\{x_{a t m}<x_{b t k}\right\}}-1 / 2\right)\right)
$$$$
+\operatorname{cov}\left(\frac{1}{\sqrt{n} n_{a}} \sum_{j=1}^{n_{b}} \sum_{i=1}^{n_{a}}\left(I_{\left\{x_{b s j}<x_{a s i}\right\}}-1 / 2\right),\right.
$$$$
\left.\sum_{d=1, d \neq a, d \neq b}^{r} \frac{1}{\sqrt{n} n_{b}} \sum_{m=1}^{n_{d}} \sum_{k=1}^{n_{b}}\left(I_{\left\{x_{d t m}<x_{b t k}\right\}}-1 / 2\right)\right)
$$$$
+\operatorname{cov}\left(\sum_{c=1, c \neq a, c \neq b}^{r} \frac{1}{\sqrt{n} n_{a}} \sum_{j=1}^{n_{c}} \sum_{i=1}^{n_{a}}\left(I_{\left\{x_{c s j}<x_{a s i}\right\}}-1 / 2\right),\right.
$$$$
\left.\frac{1}{\sqrt{n} n_{b}} \sum_{m=1}^{n_{a}} \sum_{k=1}^{n_{b}}\left(I_{\left\{x_{a t m}<x_{b t k}\right\}}-1 / 2\right)\right)
$$$$
+\sum_{c=1, c \neq a, c \neq b}^{r} \operatorname{cov}\left(\frac{1}{\sqrt{n} n_{a}} \sum_{j=1}^{n_{c}} \sum_{i=1}^{n_{a}}\left(I_{\left\{x_{c s j}<x_{a s i}\right\}}-1 / 2\right),\right.
$$$$
\left.\frac{1}{\sqrt{n} n_{b}} \sum_{m=1}^{n_{c}} \sum_{k=1}^{n_{b}}\left(I_{\left\{x_{c t m}<x_{b t k}\right\}}-1 / 2\right)\right)
$$$$
=-\frac{1}{n n_{a} n_{b}}\left[n_{a} n_{b}\left(n_{b}-1\right) \operatorname{cov}\left(F_{b s}^{o}\left(X_{a s}\right), F_{b t}^{o}\left(X_{a t}\right)\right)\right.
$$$$
\left.+n_{b} n_{a}\left(n_{a}-1\right) \operatorname{cov}\left(F_{a s}^{o}\left(X_{b s}\right), F_{a t}^{o}\left(X_{b t}\right)\right)+n_{a} n_{b} \sigma\right]
$$$$
+\sum_{c=1, c \neq a, c \neq b}^{r}\left\{\frac { 1 } { n n _ { a } n _ { b } } n _ { c } n _ { a } n _ { b } \left[-\operatorname{cov}\left(F_{a s}^{o}\left(X_{b s}\right), F_{c t}^{o}\left(X_{b t}\right)\right)\right.\right.
$$

$$
\left.\left.-\operatorname{cov}\left(F_{c s}^{o}\left(X_{a s}\right), F_{b t}^{o}\left(X_{a t}\right)\right)+\operatorname{cov}\left(F_{a s}^{o}\left(X_{c s}\right), F_{b t}^{o}\left(X_{c t}\right)\right)\right]\right\} .
$$

When $\min _{i=1,2, \ldots, r} n_{i} \longrightarrow \infty$

$$
\begin{aligned}
\left.v^{(a, b)}(s, t)\right|_{a \neq b} & \\
= & -\left[\eta_{b} \operatorname{cov}\left(F_{b s}^{o}\left(X_{a s}\right), F_{b t}^{o}\left(X_{a t}\right)\right)\right. \\
& \left.+\eta_{a} \operatorname{cov}\left(F_{a s}^{o}\left(X_{b s}\right), F_{a t}^{o}\left(X_{b t}\right)\right)\right] \\
& +\sum_{c=1, c \neq a, c \neq b}^{r} \eta_{c}\left[\operatorname{cov}\left(F_{a s}^{o}\left(X_{c s}\right), F_{b t}^{o}\left(X_{c t}\right)\right)\right. \\
& -\operatorname{cov}\left(F_{a s}^{o}\left(X_{b s}\right), F_{c t}^{o}\left(X_{b t}\right)\right) \\
& \left.-\operatorname{cov}\left(F_{c s}^{o}\left(X_{a s}\right), F_{b t}^{o}\left(X_{a t}\right)\right)\right] .
\end{aligned}
$$

III) This is a direct result from II.

\section{A.4 Empirical estimates of covariances}

For $a<b \in\{1,2, \ldots, r\}, s=1,2, \ldots, p$, denote the estimator of $\theta(a, b, s)$ by $\hat{\theta}(a, b, s)$, where

$$
\hat{\theta}(a, b, s)=\frac{1}{n_{a} n_{b}} \sum_{i=1}^{n_{a}} \sum_{j=1}^{n_{b}}\left[I_{\left\{x_{a s i}<x_{b s j}\right\}}-I_{\left\{x_{a s i}>x_{b s j}\right\}}\right] .
$$

Let the midrank $R_{a s i}^{(b)}=$ the midrank of $x_{a s i}$ among $\left\{x_{a s i}\right.$, $\left.x_{b s 1}, x_{b s 2}, \ldots, x_{b s n_{b}}\right\}, i=1,2, \ldots, n_{a}$, and the midrank $R_{b s j}^{(a)}=$ the midrank of $x_{b s j}$ among $\left\{x_{b s j}, x_{a s 1}, x_{a s 2}\right.$, $\left.\ldots, x_{a s n_{a}}\right\}, j=1,2, \ldots, n_{b}$. Then we have $\hat{F}_{a s}^{o}\left(X_{b s}\right)=$ $\frac{R_{b s j}^{(a)}-1}{n_{a}}$, and $\hat{F}_{b s}^{o}\left(X_{a s}\right)=\frac{R_{a s j}^{(b)}-1}{n_{b}}$. When $a<b$, let $P^{(a, b)}$ be the $n_{a} \times p$ matrix with $(i, s)$ element

$$
p^{(a, b)}(i s)=2 R_{a s i}^{(b)}-2-n_{b}+n_{b} \hat{\theta}(a, b, s),
$$

and $P^{(b, a)}$ be the $n_{b} \times p$ matrix with $(j, s)$ element

$$
p^{(b, a)}(j s)=2 R_{b s j}^{(a)}-2-n_{a}-n_{a} \hat{\theta}(a, b, s) .
$$

A consistent estimator of $\operatorname{cov}\left(F_{b s}^{o}\left(X_{a s}\right), F_{b t}^{o}\left(X_{a t}\right)\right)$ is then given by

$$
\begin{aligned}
\widehat{\operatorname{cov}} & \left(F_{b s}^{o}\left(X_{a s}\right), F_{b t}^{o}\left(X_{a t}\right)\right) \\
= & \frac{1}{n_{a}} \sum_{i=1}^{n_{a}}\left[\frac{R_{a s j}^{(b)}-1}{n_{b}}-\frac{1-\hat{\theta}(a, b, s)}{2}\right] \\
& \times\left[\frac{R_{a t j}^{(b)}-1}{n_{b}}-\frac{1-\hat{\theta}(a, b, t)}{2}\right] \\
= & \frac{1}{4 n_{a} n_{b}^{2}} \sum_{i=1}^{n_{a}}\left[2 R_{a s j}^{(b)}-2-n_{b}+n_{b} \hat{\theta}(a, b, s)\right] \\
& \times\left[2 R_{a t j}^{(b)}-2-n_{b}+n_{b} \hat{\theta}(a, b, t)\right] .
\end{aligned}
$$


A consistent estimate of cov $\left(F_{a s}^{o}\left(X_{b s}\right), F_{a t}^{o}\left(X_{b t}\right)\right)$ is given by

$$
\begin{aligned}
\widehat{\operatorname{cov}} & \left.F_{a s}^{o}\left(X_{b s}\right), F_{a t}^{o}\left(X_{b t}\right)\right) \\
= & \frac{1}{n_{b}} \sum_{j=1}^{n_{b}}\left[\frac{R_{b s j}^{(a)}-1}{n_{a}}-\frac{1+\hat{\theta}(a, b, s)}{2}\right] \\
& \times\left[\frac{R_{b t j}^{(a)}-1}{n_{a}}-\frac{1+\hat{\theta}(a, b, t)}{2}\right] \\
= & \frac{1}{4 n_{a}^{2} n_{b}} \sum_{j=1}^{n_{b}}\left[2 R_{b s j}^{(a)}-2-n_{a}-n_{a} \hat{\theta}(a, b, s)\right] \\
& \times\left[2 R_{b t j}^{(a)}-2-n_{a}-n_{a} \hat{\theta}(a, b, t)\right] .
\end{aligned}
$$

Then, a consistent estimator of the covariance matrix can be derived, whose elements are given by

$$
\begin{aligned}
& \left(\widehat{\operatorname{cov}}\left(F_{b s}^{o}\left(X_{a s}\right), F_{b t}^{o}\left(X_{a t}\right)\right)\right)=\frac{\left(P^{(a, b)}\right)^{\prime} P^{(a, b)}}{4 n_{a} n_{b}^{2}}, \\
& \left(\widehat{\operatorname{cov}}\left(F_{a s}^{o}\left(X_{b s}\right), F_{a t}^{o}\left(X_{b t}\right)\right)\right)=\frac{\left(P^{(b, a)}\right)^{\prime} P^{(b, a)}}{4 n_{a}^{2} n_{b}},
\end{aligned}
$$

for $a<b \in\{1,2, \ldots, r\}$.

Similarly, for $a<b<c \in\{1,2, \ldots, r\}$, we have

$$
\begin{aligned}
& \left(\widehat{\operatorname{cov}}\left(F_{b s}^{o}\left(X_{a s}\right), F_{c t}^{o}\left(X_{a t}\right)\right)\right)=\frac{\left(P^{(a, b)}\right)^{\prime} P^{(a, c)}}{4 n_{a} n_{b} n_{c}}, \\
& \left(\widehat{\operatorname{cov}}\left(F_{c s}^{o}\left(X_{a s}\right), F_{b t}^{o}\left(X_{a t}\right)\right)\right)=\frac{\left(P^{(a, c)}\right)^{\prime} P^{(a, b)}}{4 n_{a} n_{b} n_{c}}, \\
& \left(\widehat{\operatorname{cov}}\left(F_{a s}^{o}\left(X_{b s}\right), F_{c t}^{o}\left(X_{b t}\right)\right)\right)=\frac{\left(P^{(b, a)}\right)^{\prime} P^{(b, c)}}{4 n_{a} n_{b} n_{c}}, \\
& \left(\widehat{\operatorname{cov}}\left(F_{c s}^{o}\left(X_{b s}\right), F_{a t}^{o}\left(X_{b t}\right)\right)\right)=\frac{\left(P^{(b, c)}\right)^{\prime} P^{(b, a)}}{4 n_{a} n_{b} n_{c}},
\end{aligned}
$$

and

$$
\begin{aligned}
& \left(\widehat{\operatorname{cov}}\left(F_{a s}^{o}\left(X_{c s}\right), F_{b t}^{o}\left(X_{c t}\right)\right)\right)=\frac{\left(P^{(c, a)}\right)^{\prime} P^{(c, b)}}{4 n_{a} n_{b} n_{c}}, \\
& \left(\widehat{\operatorname{cov}}\left(F_{b s}^{o}\left(X_{c s}\right), F_{a t}^{o}\left(X_{c t}\right)\right)\right)=\frac{\left(P^{(c, b)}\right)^{\prime} P^{(c, a)}}{4 n_{a} n_{b} n_{c}} .
\end{aligned}
$$

\section{ACKNOWLEDGEMENTS}

We would like to thank the editor, associate editor and the anonymous reviewers for their insightful comments, which improve the manuscript substantially. We thank Dr. Tonja Nansel for providing the dietary quality and nutrients intake data to exemplify the methods. Research of Qizhai Li is partially supported by NSFC, No. 61134013. Research of Aiyi Liu is supported by the Intramural Research Program of the Eunice Kennedy Shriver National Institute of Child Health and Human Development (NICHD), National Institutes of Health (NIH), USA.

\section{Received 7 September 2012}

\section{REFERENCES}

Brunner, E., Domhof, S., and Langer, F. (2002a). Nonparametric Analysis of Longitudinal Data in Factorial Experiments. John Wiley \& Sons, INC., New Year. MR1865401

Brunner, E., Munzel, U., and Puri, M. L. (2002b). The multivariate nonparametric Behrens-Fisher problem. Journal of Statistical Planning and Inference 108 37-53. MR1947390

Genz, A. (1993). Comparison of methods for the computation of multivariate normal probabilities. Computing Science and Statistics $\mathbf{2 5}$ 400-405.

Genz, A. and Bretz, F. (2009). Computation of Multivariate Normal and $t$ Probabilities. Lecture Notes in Statistics 195. Springer-Verlag, Heidelberg. MR2840595

Huang, P., Tilley, B. C., Woolson, R. F., and Lipsitz, S. (2005). Adjusting O'Brien's test to control type I error for the generalized nonparametric Behrens-Fisher problem. Biometrics 61532 539. MR2140925

KNIP, M. (2009). Diet, gut, and type 1 diabetes: Role of wheat-derived peptides? Diabetes 58 1723-1724.

LiU, A., LI, Q., LiU, C., YU, K. and Yu, K. F. (2010). A rank-based test for comparison of multidimensional outcomes. Journal of the American Statistical Association 105 578-587. MR2724843

Nansel, T. R., Gellar, L., and McGill, A. (2008). Effect of varying glycemic index meals on blood sugar control assessed with continuous glucose monitoring in youth with type 1 diabetes on basal-bolus insulin regimens. Diabetes Care 31 695-697.

O'Brien, P. C. (1984). Procedures for comparing samples with multiple endpoints. Biometrics 40 1079-1087. MR0786180

Tong, Y. L. (1990). The Multivariate Normal Distribution. Springer, New York. 105 578-587. MR1029032

Zhengbang Li

School of Mathematics and Statistics

Central China Normal University

Wuhan 430079

China

E-mail address: lizhengbang@mail.ccnu.edu.com

Aiyi Liu

Eunice Kennedy Shriver National Institute of Child Health and Human Development

National Institutes of Health

Bethesda 20892

USA

E-mail address: liua@mail.nih.gov 
Zhaohai Li

Department of Statistics

George Washington University

Washington, DC 20052

USA

Biostatistics Branch

Division of Cancer Epidemiology and Genetics

National Cancer Institute

National Institutes of Health

Bethesda 20892

USA

E-mail address: zli@gwu.edu
Qizhai Li

Academy of Mathematics and Systems Science

Chinese Academy of Sciences

Beijing 100190

China

E-mail address: liqz@amss.ac.cn url: www.statsci.amss.ac.cn/QZLiPage/ LiQizhaiHomepage.htm 